\title{
Video-augmented vs standard consent in an early ICU cycling feasibility trial: a randomized embedded recruitment trial
}

\section{[version 1; peer review: 1 approved, 1 approved with}

\section{reservations]}

\author{
Michelle E. Kho (D1,2, Mark Duffett (iD)3,4, France J. Clarke4, Melissa Shears5, \\ Alexander J. Molloy2, Deborah J. Cook ${ }^{4,6}$ \\ ${ }^{1}$ School of Rehabiltation Science, McMaster University, Hamilton, ON, Canada \\ ${ }^{2}$ Department of Physiotherapy, St. Joseph's Healthcare Hamilton, Hamilton, ON, Canada \\ ${ }^{3}$ Department of Pediatrics, McMaster University, Hamilton, ON, Canada \\ ${ }^{4}$ Department of Health Research Methods, Evidence, and Impact, McMaster University, Hamilton, ON, Canada \\ ${ }^{5}$ Department of Anesthesiology and Pain Medicine, University of Alberta, Edmonton, AB, Canada \\ ${ }^{6}$ Department of Medicine, McMaster University, Hamilton, ON, Canada
}

\author{
V1 First published: 24 Jan 2020, 9:45 \\ https://doi.org/10.12688/f1000research.21750.1 \\ Latest published: 24 Jan 2020, 9:45 \\ https://doi.org/10.12688/f1000research.21750.1
}

\section{Abstract}

Background: In a trial of early in-bed cycling in critically ill patients, a video demonstrating use of the cycle in addition to verbal description may improve satisfaction with the informed consent process for all persons involved.

Methods: A convenience sample of in-person consent encounters for enrolment in TryCYCLE (NCT01885442), a 33-patient pilot study of inbed cycling with mechanically ventilated patients in an intensive care unit, were recruited. In this study within a trial, using concealed allocation, we randomized consent encounters to a Video or Standard consent approach. Those in the Video group viewed a 2-minute video of a model using in-bed cycling plus the routine verbal description of the study. The Standard group received the routine verbal description only. Patients and/or substitute decision makers (SDMs) were blinded to the study purpose. After each encounter, patients and/or SDMs and the research coordinator submitted written satisfaction and comfort ratings using 7-point scales (higher scores better). We documented consent outcomes and analyzed between group differences with independent group t-tests.

Results: We randomized 14 encounters (6 Video, 8 Standard). Ten completed questionnaires (5 in each group) demonstrated no difference in patient and/or SDM satisfaction or comfort between Video or Standard (mean [standard deviation] Satisfaction: 6.8[0.45] vs. 7.0[0] vs. $p=0.37$; Comfort: $7.0[0]$ vs. $7.0[0], p>0.99$ ). The research

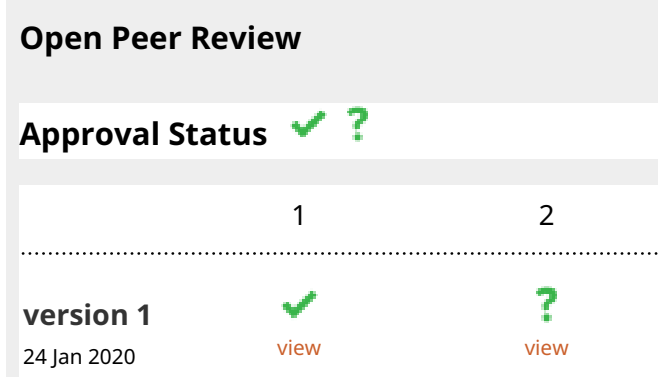

1. David J. Torgerson ID, University of York, York, UK

2. Thierry Boulain (iD), Centre Hospitalier

Régional d'Orléans, Orléans, France

Any reports and responses or comments on the article can be found at the end of the article. 
coordinator evaluated all randomized encounters, with no differences between Video or Standard (Satisfaction: 7.0[0] vs. 6.9[0.35], $p=0.41$; Comfort: 6.7[0.52] vs. 6.9[0.35], $p=0.39$ ). All 14 consent encounters enrolled in TryCYCLE.

Conclusions: Patient and/or SDM satisfaction and comfort with consent was very high for both the Video and Standard approaches. Further research, including use of videos to portray different study interventions, is needed, including analysis of patient and/or SDM satisfaction, comfort, comprehension, and consent rates.

Registration for host trial: ClinicalTrials.gov, NCT01885442, registered on June 25, 2013

\section{Keywords}

Evidence-based Medicine, Randomized controlled Trials, Research design, Informed consent, Clinical trials, Audiovisual aids, mechanical ventilation, cycle ergometry, Studies within a trial

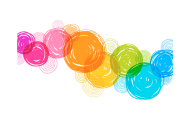

This article is included in the Studies Within A Trial (SWAT) collection.

Corresponding author: Michelle E. Kho (khome@mcmaster.ca)

Author roles: Kho ME: Conceptualization, Data Curation, Formal Analysis, Funding Acquisition, Investigation, Methodology, Project Administration, Resources, Supervision, Validation, Visualization, Writing - Original Draft Preparation; Duffett M: Methodology, Writing Original Draft Preparation, Writing - Review \& Editing; Clarke FJ: Investigation, Methodology, Validation, Writing - Review \& Editing; Shears M: Data Curation, Writing - Review \& Editing; Molloy AJ: Methodology, Validation, Writing - Review \& Editing; Cook DJ: Conceptualization, Investigation, Methodology, Writing - Review \& Editing

Competing interests: No competing interests were disclosed.

Grant information: MK and DC are each funded by a Canadian Institutes for Health Research Canada Research Chair. The TryCYCLE study was funded by the Canadian Institutes of Health Research Institute of Musculoskeletal Health and Aging New Investigators Bridge Funding [131584].

The funders had no role in study design, data collection and analysis, decision to publish, or preparation of the manuscript.

Copyright: ( $) 2020$ Kho ME et al. This is an open access article distributed under the terms of the Creative Commons Attribution License, which permits unrestricted use, distribution, and reproduction in any medium, provided the original work is properly cited.

How to cite this article: Kho ME, Duffett M, Clarke FJ et al. Video-augmented vs standard consent in an early ICU cycling feasibility trial: a randomized embedded recruitment trial [version 1; peer review: 1 approved, 1 approved with reservations]

F1000Research 2020, 9:45 https://doi.org/10.12688/f1000research.21750.1

First published: 24 Jan 2020, 9:45 https://doi.org/10.12688/f1000research.21750.1 


\section{Introduction}

In-bed cycling is a novel critical care rehabilitation intervention that could improve patients' physical function ${ }^{1}$. In-bed cycling is not commonly used in the intensive care unit (ICU) ${ }^{2}$ and is likely unfamiliar to many patients and their substitute decision makers (SDMs). A video demonstrating the use of the cycle in addition to verbal informed consent may improve their satisfaction and comfort with the consent process. The purpose of this study within a trial (SWAT) was to determine the effect of video-augmented versus standard informed consent on patient and SDM satisfaction and comfort.

\section{Methods}

The study protocol is available as Extended data ${ }^{3}$.

\section{Study design}

We included a convenience sample of consent encounters in TryCYCLE (NCT01885442), a 33-patient, single-centered, safety and feasibility study of in-bed cycling in critically ill patients ${ }^{4}$. The TryCYCLE study required a priori informed consent. Patients and SDMs who were approached for in-person informed consent for TryCYCLE in our mixed medical-surgical ICU (St Joseph's Healthcare ICU) in Hamilton, ON were eligible for this substudy. We excluded encounters where initial consent was received by phone, as respondents could not view the video as part of their initial deliberations.

\section{Randomization}

Using concealed allocation with variable undisclosed block size, we randomized consent encounters to receive augmented video consent in addition to verbal consent discussion (Video) or verbal consent discussion only (Standard) in a 1:1 ratio. To maintain allocation concealment, we used third-party assignment. An individual not otherwise involved in the study (MD) prepared a computer-generated randomization sequence and provided the group assignment immediately prior to each consent encounter.

\section{Intervention}

The research coordinator used a tablet computer to show and describe how an ICU patient would bike in the TryCYCLE study. The 2-minute video included footage of a model on the bike and an ICU patient biking while mechanically ventilated. The research coordinator also provided a verbal description of the research study, which was not scripted to allow for spontaneous exchange. The research coordinator and patient and/or SDM had the consent encounter guided by the usual principles of informed consent for ICU studies previously published $^{5}$. The video did not include sound.

Comparison: Verbal description only.

\section{Outcomes}

Our primary outcomes were patient and/or SDM satisfaction and comfort with the consent encounter. Secondary outcomes included patient and/or SDM perception of video helpfulness, and research coordinator assessments of satisfaction with the consent process, their own comfort with the consent encounter, patient and/or SDM study comprehension, and the number and perceived difficulty of questions asked by patient and/or SDM. Respondents assessed outcomes after the consent encounter using 7-point Likert-type scales, with higher scores representing more favorable ratings (see Extended data $^{3}$ ). We invited patients and/or SDMs to return the 1-page, selfadministered questionnaire within 24 hours in a pre-addressed, sealable envelope. The research coordinator documented consent outcome and duration of the encounter.

\section{Blinding}

Patients and SDMs were not aware of the comparison study at the time of the consent encounter. Both groups received the same consent forms for the TryCYCLE study ${ }^{3}$. The analyst was blinded to treatment group.

\section{Sample size and data analysis}

We did not calculate a sample size a priori for this SWAT. We reported continuous variables as mean (standard deviation [SD]) and categorical values as count (percent). We used Student's t-tests to compare the results of the two groups, Pearson's Chi Square for categorical comparisons, and $\mathrm{p}=0.05$ as the criterion for statistical significance. We conducted all analyses with SPSS version 25.0 (IBM, Armonk, New York).

\section{Ethics}

This study, and the host study (TryCYCLE) were approved by the Hamilton Integrated Research Ethics Board (HIREB 13-173), which waived the need for consent for this substudy. Study participants could opt-out of this substudy by declining to return the follow-up questionnaire.

\section{Results}

We randomized 14 of the 37 consent encounters for TryCYCLE between October 2013 and August 2014: 6 to Video and 8 to Standard (Figure 1). Table 1 summarizes the characteristics of the convenience sample in this study. We received 10 completed questionnaires (5 from each group), which contributed to estimates of effect. We report all comparisons as Video vs. Standard. Patient and/or SDM rating of satisfaction was not different between groups, the mean [SD] was 6.8 [0.5] vs. 7.0 [0] $(\mathrm{p}=0.37)$. Patient and/or SDM rating of comfort was the same for both groups (7.0 [0], $\mathrm{p}>0.99)$. Patient and/or SDM rating of video helpfulness was 6.6 [0.54]. All 14 (100\%) patients and/or SDMs provided consent to participate in the TryCYCLE study (the overall consent rate for TryCYCLE was 91.9\%).

Of the 14 consent encounters rated by the research coordinator, satisfaction (7.0 [0] vs. $6.9[0.35], \mathrm{p}=0.41)$ and comfort (6.7 [0.52] vs. 6.9 [0.35], $\mathrm{p}=0.39)$ were similar. There was no difference in perceived patient and/or SDM understanding of the research study (6.67 [0.51] vs. 7.0 [0] vs., $\mathrm{p}=0.11)$, number of questions asked (1-5 questions: $6(75.0 \%)$ vs. $5(83.3 \%), \mathrm{p}=0.66)$, nor perceived difficulty of clarifying questions $(2.67$ [1.86] vs. 


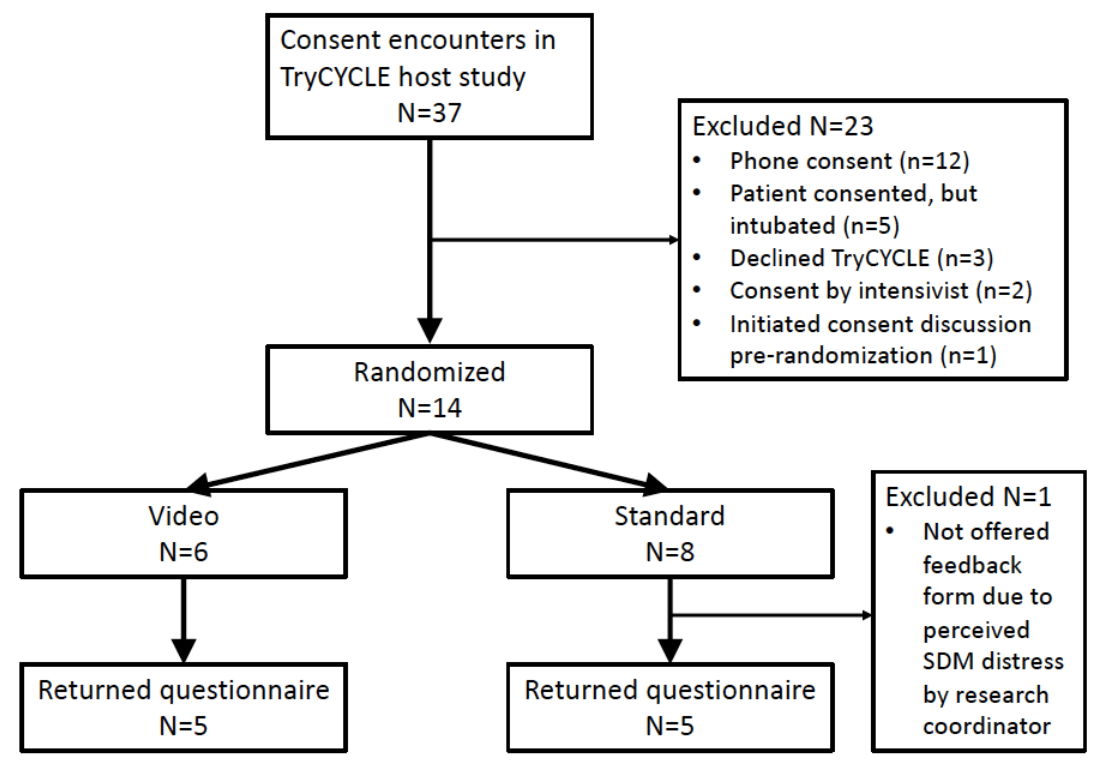

Figure 1. Consent encounter flow diagram for this study. Of the 23 non-randomized patients who had consent encounters, 12 occurred by phone, 5 were in-person by intubated patients who were unable to complete the feedback form, 3 declined participation in the host study and these consent encounters occurred without the option for enrolment in the SWAT, 2 in-person consents were obtained by a consultant intensivist, and in 1 instance we already initiated the consent discussion before consideration of randomization in this trial. Post-randomization, 1 person was not offered the feedback form due to perceived emotional distress by the research coordinator.

Table 1. Participant characteristics involved in the consent encounter. SDM= substitute decision maker.

\begin{tabular}{|l|r|r|r|}
\hline $\mathbf{n}(\mathbf{\%})$ & Overall $(\mathbf{n = 1 4})$ & Video + Verbal $\mathbf{( n = 6 )}$ & Verbal $\mathbf{( n = 8 )}$ \\
\hline Female & $8(57 \%)$ & $2(33 \%)$ & $5(63 \%)$ \\
\hline Included in video substudy: & & & \\
\hline SDM & $11(79 \%)$ & $4(66 \%)$ & $7(88 \%)$ \\
Patient & $1(7 \%)$ & $1(17 \%)$ & 0 \\
SDM and Patient & $2(14 \%)$ & $1(17 \%)$ & $1(3 \%)$ \\
\hline Consented for TryCYCLE & $17(100 \%)$ & $6(100 \%)$ & $8(100 \%)$ \\
\hline
\end{tabular}

$1.86[1.4], \mathrm{p}=0.40)$. The encounter duration was not different (18.3[14.7] vs. $13.3[3.7]$ minutes, $\mathrm{p}=0.20)$.

\section{Discussion}

In this small randomized study of video-augmented versus standard consent for in-bed cycling in the ICU, there was no difference in satisfaction or comfort between groups. Our results are similar to previous studies included in a systematic review of audiovisual plus standard versus standard informed consent, identifying no difference in consent rate between groups ${ }^{6}$. Given the universally high ratings for satisfaction and comfort by consent recipients, we hypothesize that video-augmented consent could be useful for describing more complex interventions or facilitating conversations when research centres or coordinators are new to conducting clinical trials. Involving patient-family advisory groups may help guide use and content of video adjuncts for future consent encounters.

Our study also contributes to global efforts to study efficiencies in trial conduct through SWATs ${ }^{7}$. It directly addresses Priority 4 (What are the best approaches for designing and delivering information to members of the public who are invited to take part in a randomized trial) of the top 10 trial recruitment issues informed by stakeholders, including members of the public, front line clinical and research staff, trial principal investigators, and funders ${ }^{8}$.

Our study has limitations, principally the small sample size and significant expertise in consent discussions from a single research coordinator with 20 years of experience in critical care 
studies. We did not measure consent recipient knowledge. Our study also has several strengths. Patients and/or SDMs were blinded to the Video vs Standard comparison study. We evaluated practical aspects of the consent process, focusing on outcomes we anticipate are important to patients and SDMs. We addressed gaps in previous research by measuring SDM and clinical researcher satisfaction, and time to administer the videoaugmented consent ${ }^{6}$.

\section{Conclusions}

Overall, patient, SDM, and research coordinator satisfaction and comfort with consent was very high for both the videoaugmented and standard approaches. Further research, including use of videos to portray different study interventions, is needed, including analysis of patient and/or SDM satisfaction, comfort, comprehension, and consent rates.

\section{Data availability}

\section{Underlying data}

Since the intervention video includes an identifiable critically ill patient, and permission to publish this was not obtained from the patient, patient's family or the research ethics board, this cannot be shared.

Open Science Framework: Video-augmented vs. standard consent in an early ICU cycling feasibility trial: a randomized embedded recruitment trial, https://doi.org/10.17605/OSF. $\mathrm{IO} / 4 \mathrm{SPGA}^{3}$
This project contains the following underlying data:

- TryCYCLE Video RCT data-SDM CRF.csv: data corresponding to data collection forms for patient/SDM

- TryCYCLE Video RCT data-RC CRF.csv: data corresponding to data collection forms for research coordinator

\section{Extended data}

Open Science Framework: Video-augmented vs. standard consent in an early ICU cycling feasibility trial: a randomized embedded recruitment trial, https://doi.org/10.17605/OSF. $\mathrm{IO} / 4 \mathrm{SPGA}^{3}$

This project contains the following extended data:

- TryCYCLE Video consent study protocol.docx

- TryCYCLE Video consent data collection forms.docx

Reporting guidelines

Open Science Framework: CONSORT checklist for 'VideoAugmented vs Standard Consent in an Early ICU Cycling Feasibility Trial: A Randomized Embedded Recruitment Trial', https://doi.org/10.17605/OSF.IO/4SPGA ${ }^{3}$

Data are available under the terms of the Creative Commons Zero "No rights reserved" data waiver (CC0 1.0 Public domain dedication).
1. Burtin C, Clerckx B, Robbeets C, et al.: Early exercise in critically ill patients enhances short-term functional recovery. Crit Care Med. 2009; 37(9): 2499-505. PubMed Abstract | Publisher Full Text

2. Koo KK, Choong K, Cook DJ, et al.: Early mobilization of critically ill adults: a survey of knowledge, perceptions and practices of Canadian physicians and physiotherapists. CMAJ Open. 2016; 4(3): E448-E54. PubMed Abstract | Publisher Full Text | Free Full Text

3. Kho M: Video-augmented vs. standard consent in an early ICU cycling feasibility trial: a randomized embedded recruitment trial. 2020. http://www.doi.org/10.17605/OSF.IO/4SPGA

4. Kho ME, Molloy AJ, Clarke FJ, et al:: TryCYCLE: A Prospective Study of the Safety and Feasibility of Early In-Bed Cycling in Mechanically Ventilated Patients. PLoS One. 2016; 11(12): e0167561. PubMed Abstract | Publisher Full Text | Free Full Text

5. Smith OM, McDonald E, Zytaruk N, et al:: Enhancing the informed consent process for critical care research: strategies from a thromboprophylaxis trial. Intensive Crit Care Nurs. 2013; 29(6): 300-309.

PubMed Abstract | Publisher Full Text

6. Synnot A, Ryan R, Prictor M, et al:: Audio-visual presentation of information fo informed consent for participation in clinical trials. Cochrane Database Syst Rev. 2014; (5): CD003717.

PubMed Abstract | Publisher Full Text | Free Full Text

7. Treweek S, Bevan S, Bower P, et al.: Trial Forge Guidance 1: what is a Study Within A Trial (SWAT)? Trials. 2018; 19(1): 139. PubMed Abstract | Publisher Full Text | Free Full Text

8. Healy P, Galvin S, Williamson PR, et al:: Identifying trial recruitment uncertainties using a James Lind Alliance Priority Setting Partnership - the PRioRiTy (Prioritising Recruitment in Randomised Trials) study. Trials. 2018 19(1): 147.

PubMed Abstract | Publisher Full Text | Free Full Text 


\section{Open Peer Review}

\section{Current Peer Review Status:}

\section{Version 1}

Reviewer Report 01 June 2020

https://doi.org/10.5256/f1000research.23976.r63783

(C) 2020 Boulain T. This is an open access peer review report distributed under the terms of the Creative Commons Attribution License, which permits unrestricted use, distribution, and reproduction in any medium, provided the original work is properly cited.

\section{Thierry Boulain}

Service de Médecine Intensive Réanimation, Centre Hospitalier Régional d'Orléans, Orléans, France

In this study within a trial, the authors included surrogate decision-makers (SDM) approached for potential consent to the enrollment of patients in a randomized trial. The aim of this trial within a trial was to compare the satisfaction and comfort of the SDM (and of patients, but finally very few patients were included) regarding the consent encounter, between two groups who were randomly allocated to video-augmented consent encounter or standard consent encounter. The main findings are that SMD reported similar satisfaction and comfort scores in both groups. Finally, only 5 subjects in each group could be studied.

The obvious, main weakness of this study is its very small sample size.

This has several consequences: First, estimates of outcome measures (satisfaction and comfort scores) are imprecise; Second, comparison tests between groups have very low power; Third, the authors conclude that comfort score was very high in both groups. Indeed all SDM gave a score of 7 on the 7-point Likert scale. Imagine the "very high score" was defined as a score of 7. In the convenience sample studied, the proportion of patients with very high score was $100 \%(5 / 5)$ in each group. This only tells us that the proportion of high score in the population is between $57 \%$ and $100 \%$ (95\% Wilson confidence interval) (and perhaps close to $57 \%$ in one group and close to $100 \%$ in the other group). Is a very high score proportion of $57 \%$ satisfactory? This is a qualitative judgment, but I would say no.

Scores on Likert scales are presented as means and SD and compared using parametric test. This assumes that the Likert scale is a homogeneous continuous scale where the distance or the increase in comfort is the same between each point, and that the distance between, say point 4 and point 6 , is two times the distance between point 1 and point 2 . This assumption is likely not true. For this reason, I would give median and percentiles to report scores and perform betweengroup comparison using a nonparametric test (Mann-Withney $U$ test).

Also, given the small samples studied, presenting all individual data, in boxplots, for example, would give an honest picture of what was observed. 
As explained above, and although the authors were cautious in referring only to "high scores" in their conclusion, even this conclusion is not fully supported by the observed data.

Is the work clearly and accurately presented and does it cite the current literature? Yes

Is the study design appropriate and is the work technically sound?

Yes

Are sufficient details of methods and analysis provided to allow replication by others? Yes

If applicable, is the statistical analysis and its interpretation appropriate? Partly

Are all the source data underlying the results available to ensure full reproducibility? No source data required

Are the conclusions drawn adequately supported by the results? Partly

Competing Interests: No competing interests were disclosed.

Reviewer Expertise: Intensive care medicine, clinical research

I confirm that I have read this submission and believe that I have an appropriate level of expertise to confirm that it is of an acceptable scientific standard, however I have significant reservations, as outlined above.

Reviewer Report 28 January 2020

https://doi.org/10.5256/f1000research.23976.r59119

(c) 2020 Torgerson D. This is an open access peer review report distributed under the terms of the Creative Commons Attribution License, which permits unrestricted use, distribution, and reproduction in any medium, provided the original work is properly cited.

\section{David J. Torgerson}

York Trials Unit, Department of Health Sciences, University of York, York, UK

This is an interesting SWAT, which despite its small size is of interest. A few suggestions to improve the reporting:

Have you registered the SWAT with the Northern Ireland Hub for Trials Methodology Research (

https://www.qub.ac.uk/sites/TheNorthernIrelandNetworkforTrialsMethodologyResearch/SWATSWARInfo 
this will allow others to replicate the study as this is obviously required.

The reporting of the means should also include the differences and $95 \%$ confidence intervals of the differences.

You used block randomisation - what block sizes were used?

With a tiny sample size, I think some qualitative outcomes, if collected, would enhance the findings of the study.

Is the work clearly and accurately presented and does it cite the current literature? Yes

Is the study design appropriate and is the work technically sound?

Yes

Are sufficient details of methods and analysis provided to allow replication by others? Partly

If applicable, is the statistical analysis and its interpretation appropriate?

Partly

Are all the source data underlying the results available to ensure full reproducibility? Partly

Are the conclusions drawn adequately supported by the results?

Yes

Competing Interests: No competing interests were disclosed.

Reviewer Expertise: Interest in SWATS and their conduct as well as being the Director of Trials Unit I confirm that I have read this submission and believe that I have an appropriate level of expertise to confirm that it is of an acceptable scientific standard. 
The benefits of publishing with F1000Research:

- Your article is published within days, with no editorial bias

- You can publish traditional articles, null/negative results, case reports, data notes and more

- The peer review process is transparent and collaborative

- Your article is indexed in PubMed after passing peer review

- Dedicated customer support at every stage

For pre-submission enquiries, contact research@f1000.com 